\title{
Use Of Yttrium-90 Microspheres In Patients With Advanced Hepatocellular Carcinoma \& Portal Vein Thrombosis
}

\author{
Andrea L. Tsai, A.M. \\ University of North Carolina School of Medicine \\ Charles T. Burke, M.D. \\ University of North Carolina School of Medicine \\ Andrew S. Kennedy, M.D., FACRO \\ Wake Radiology Oncology \\ Dominic T. Moore, Ph.D. \\ University of North Carolina School of Medicine
}

Matthew A. Mauro, M.D., F.A.C.R., F.S.I.R., F.A.H.A.

University of North Carolina School of Medicine

Robert D. Dixon, M.D.

University of North Carolina School of Medicine

Joseph M. Stavas, M.D.

University of North Carolina School of Medicine

\section{Stephen A. Bernard, M.D.}

University of North Carolina School of Medicine

\author{
Amir H. Khandani, M.D. \\ University of North Carolina School of Medicine \\ Bert H. O'Neil, M.D. \\ University of North Carolina School of Medicine
}

\begin{abstract}
Purpose-Patients with portal vein thrombosis (PVT) and hepatocellular carcinoma (HCC) have limited treatment options due to increased disease burden and diminished hepatic perfusion. 90Ymicrospheres may be better tolerated than chemoembolization in these patients. Here we review the safety and efficacy of 90Y-microsphere use for HCC with major PVT.

Materials and Methods-A retrospective review of HCC with main $(n=10)$ or first $(n=12)$ branch PVT treated with 90Y-microspheres $(\mathrm{n}=22)$ was conducted. CLIP scores ranged from 2 to 5 with $18 \%$ scoring 4 or greater. Response was determined 8-12 weeks following treatment using magnetic
\end{abstract}

(C) 2010 The Society of Interventional Radiology. Published by Elsevier Inc. All rights reserved

Publisher's Disclaimer: This is a PDF file of an unedited manuscript that has been accepted for publication. As a service to our customers we are providing this early version of the manuscript. The manuscript will undergo copyediting, typesetting, and review of the resulting proof before it is published in its final citable form. Please note that during the production process errors may be discovered which could affect the content, and all legal disclaimers that apply to the journal pertain.

I certify that I and my co-authors have complied with the policy on financial disclosure outlined in the Information for Authors located on the JVIR Web Site and that we have no financial relationships or conflicts of interest.

The material was not presented at an SIR annual meeting. 
resonance or computed tomography and RECIST criteria. Overall survival was estimated by the Kaplan-Meier method.

Results - 32 treatments (26 glass, 6 resin) were administered to 22 patients. Common grade 1-2 toxicities included abdominal pain (38\%), nausea (28\%), fatigue (22\%). Four post-therapy hospitalizations occurred, all $<48 \mathrm{hrs}$ in duration. 1 death occurred 10 days following therapy Response data: 2 partial responses, progressive disease $42 \%$, stable disease $50 \%$ of treatments. Median overall survival (OS) was 7 months from time of initial 90Y-microsphere treatment. ChildPugh A patients had a median OS of 7.7 months; $B$ and $C=2.7$ months $(p=0.01)$. Median OS for CLIP scores 2-3 was 7 months versus 1.3 months for scores $4-5$ ( $\mathrm{p}=0.04)$.

Conclusions-90Y-microspheres are tolerated in patients with HCC and major PVT. Compared with chemoembolization, rates of severe adverse events appear low. Radiographic response rates are low. Median OS of 7 months is promising and warrants further study versus systemic therapy.

\section{INTRODUCTION}

Hepatocellular carcinoma (HCC) is one of the most common cancers worldwide, with an estimated incidence of over 600,000 per year (1). Up to $40 \%$ of patients with HCC develop portal vein thrombosis (PVT), which poses a challenging treatment dilemma (2). The prognosis for patients with PVT in the major branches is poor (3-5), and optimal treatment strategies are unclear. Palliative techniques such as transarterial embolization or chemoembolization are commonly used for the treatment of advanced HCC; however, patients with PVT may run a greater risk of hepatic decompensation following embolic therapy due to diminished portal venous blood flow, which compensates to maintain oxygenation of the liver when the arterial circulation is interrupted. Because of this, embolic techniques are often contraindicated in those with major PVT, or are limited to superselective techniques in patients with segmental or incomplete PVT (6-7). Additionally, the value of embolic therapies in the population of patients with PVT is unproven at this time.

The use of glass or resin yttrium-90 (90Y) microspheres is a relatively recent development in the treatment of HCC (8-9). Published evidence of efficacy and safety with the use of microspheres in patients with HCC and PVT is limited. Yttrium-90 microspheres are smaller in caliber than typical embolic particles, and are delivered to a specific radiation dose rather than to stasis. Therefore they are hypothesized to exert less of an ischemic effect compared to other methods of embolization. Lack of an embolic effect of $90 \mathrm{Y}$-microspheres has been further demonstrated by reports showing a decreased incidence of post-embolization syndrome following transarterial administration of 90Y-microspheres compared with chemoembolization or transarterial embolization (10-11). Because the primary therapeutic mechanism of $90 \mathrm{Y}$-microspheres is local radiation as opposed to ischemia, it has been postulated that $90 \mathrm{Y}$-microspheres may be better tolerated than traditional embolization or chemoembolization in patients with PVT. The purpose of this study was to test this hypothesis by retrospectively assessing $90 \mathrm{Y}$-microsphere use in a cohort of patients with advanced HCC and main or complete first branch PVT.

Here we report the results of 22 patients treated with either glass or resin $90 \mathrm{Y}$-microspheres. Though prior studies have reported use of 90Y-microspheres in HCC with PVT, the amount of safety, efficacy and outcomes data available is limited to subgroup analyses from one phase II trial (12-13). Our study was carried out specifically in patients with significant PVT. To that end we have analyzed the toxicity, response, and survival of patients with HCC and PVT receiving 90Y-microspheres. 


\section{MATERIALS AND METHODS}

\section{Patient Selection}

This retrospective review includes patients $(\mathrm{n}=22)$ with confirmed HCC and main or first branch PVT. Typically at our institution, patients with HCC without PVT or with PVT of only a secondary branch of the portal venous system are treated with chemoembolization. At the time 90Y-microspheres became available, we decided to utilize this therapeutic option specifically in patients who were not felt to be good candidates for chemoembolization, or patients whose tumors had progressed after chemoembolization (specifically those who had developed PVT in conjunction with progression of disease). Patients treated with glass spheres were treated under an Institutional Review Board (IRB)-approved protocol that did not specify tumor burden or portal vein status. Separate IRB approval was obtained to review the patient records for this study without need for additional consent. All patients provided written informed consent for treatment with $90 \mathrm{Y}$-microspheres and for collection of data for research purposes.

All patients were discussed for suitability for 90Y-microspheres by a multidisciplinary tumor board consisting of hepatologists, transplant surgeons, interventional radiologists and a medical oncologist. Eligibility for treatment with $90 \mathrm{Y}$-microspheres was based on: age $\geq 18$ years, Eastern Cooperative Oncology Group (ECOG) performance status $\leq 2$, confirmed diagnosis of surgically unresectable HCC (biopsy proven or alpha-fetoprotein, AFP > 400) and first or main branch PVT as determined by computed tomography (CT) or magnetic resonance (MR) imaging. Pertinent criteria for patient exclusion included: pregnancy, uncontrolled ascites, encephalopathy, and coagulopathy. Significantly abnormal pretreatment laboratory findings (serum creatinine $\geq 2.0 \mathrm{mg} / \mathrm{dL}$ or bilirubin $>2 \times$ upper limits of normal, ULN) were relative contraindications for treatment. All treated patients were evaluated to confirm an acceptable risk of shunting of 90Y-microspheres to the lungs ( $<30$ Gy or hepatopulmonary shunt $<20 \%$ by tagged albumin scan) and no detectable flow to other gastrointestinal organs. Four of the 19 patients receiving glass microspheres and one of the four patients receiving resin microspheres required embolization of aberrant gastric arteries prior to treatment in order to allow safe delivery of the $90 \mathrm{Y}$-microspheres.

Clinical and biochemical data were obtained at baseline and 1 to 4 weeks following treatment. A 30 day follow-up period from treatment was used as the cutoff for the safety and toxicity analysis. Toxicities occurring beyond this time point can be related to radiation-induced liver disease (14); however only acute toxicities were evaluated in this study.

\section{Treatment Planning and Delivery of 90Y-microspheres}

All patients had an initial medical history and physical examination. The baseline treatment planning work-up included CT or MR imaging of the liver, technetium- $99 \mathrm{~m}$ macro-aggregated albumin (99mTc-MAA) scan, and AFP level. Selective celiac and superior mesenteric arteriography was performed with late-phase imaging of the portal venous system to evaluate vascular anatomy, patency of the portal vein and the direction of portal flow. Treatment planning and administration of $90 \mathrm{Y}$-microspheres were performed according to previously published guidelines for use of glass (15) or resin (16) 90Y-microspheres. Glass microsphere activity is selected as an intended dose in Gray (Gy) as determined by the mass of the tumor and liver and uses MIRD phantom calculations (17-18). Resin microsphere activity is instead planned using a combination of the patient's liver volume, tumor volume, and body surface area $(16,19)$.

Liver CT and MR images were reviewed with special attention to the volume of the liver, degree and distribution of tumor infiltration and patency of the portal vein. If a patient was 
deemed a suitable candidate for 90Y-microspheres treatment based on available information, visceral arteriography and 99mTc-MAA scanning were performed to define arterial anatomy and evaluate the amount of lung shunt, gastrointestinal flow, and PVT. The amount of activity required for the target dose of 90Y-microspheres was calculated Patients were considered eligible for 90Y-microsphere treatment if the lung shunt would result in an estimated dose of less than $30 \mathrm{~Gy}$ ( $16.5 \mathrm{mCi} ; 610.5 \mathrm{GBq})$ to the lungs, there was no observable un-correctable gastrointestinal flow, and liver reserve and tumor vascular anatomy were judged to be suitable for treatment. In strict aseptic conditions and following institutional radiation safety guidelines, the calculated activity of $90 \mathrm{Y}$-microspheres was administered selectively to the target hepatic lobe by intra-arterial infusion at low pressure (20-40 pounds per square inch), according to the manufacturer's instructions. Treatment was delivered on an outpatient basis, where patients were observed in recovery for 4 to 6 hours and were discharged to home when they were clinically stable and without complaints.

Patients with bilobar disease that received glass microspheres were scheduled for treatment of the opposite lobe 30-60 days after initial treatment, according to the original treatment plan. Patients treated with resin microspheres who required retreatment were treated at least 90 days from the first treatment.

To determine eligibility for re-treatment, patients had a repeat treatment work-up, as performed before initial treatment. At the time of re-treatment evaluation, consideration of the total dose to the lungs was based on the cumulative dose of all treatments and could not exceed an estimated cumulative dose of $50 \mathrm{~Gy}$ as calculated by the calculated dose to the lung from the first treatment and the estimated lung shunt fraction from the repeat 99mTc-MAA scan (2021). In patients with complex tumor presentations who required more than two treatments to achieve tumor coverage, or in patients being retreated after progression, the safety re-evaluation was repeated before each treatment and the calculation of cumulative lung dose included all previous treatments.

\section{Data Collection}

Data comprised information on demographics, tumor characteristics and outcomes. Baseline evaluations taken prior to therapy were used to calculate Child-Pugh and Cancer of the Liver Italian Program (CLIP) scores (22). Liver function tests were collected prior to therapy and 7 to 30 days after therapy. Outcomes data after treatment included treatment-related adverse events (AEs), imaging response and overall survival. All adverse events were classified and coded for severity using the Common Terminology Criteria for Adverse Events (CTCAE) version 3.0. Response was determined approximately $8-12$ weeks following treatment using MR (whenever possible) or CT scans and evaluated using the new international criteria proposed by the Response Evaluation Criteria in Solid Tumors (RECIST) Committee (23). Patients were classified as positive for intra-hepatic disease progression based on comparative evaluation of pre- and post-treatment CT or MR images. All measurable (non-infiltrative) tumor lesions per patient were documented; however, only changes in the largest diameter (uni-dimensional measurement) tumor lesion was used to determine response, per RECIST criteria. Patients were considered to have intra-hepatic disease progression if there was visual evidence of increasing lesion size or new lesion development.

\section{Statistical Methods}

The Kaplan-Meier (or product limit) method was used to estimate survival curves. Overall survival (OS) has been defined as the time from the date of the start of treatment to the date of death (event) or the date of last contact (censored). The log-rank test was used to test for significant differences between estimated the survival curves. Exact $95 \%$ confidence intervals were calculated for each proportion of interest (and reported as percentages). Statistical 
analyses were performed with SAS statistical software, version 9.2, SAS Institute Inc., Cary, NC.

\section{RESULTS}

Thirty-two treatments ( 26 glass, 6 resin spheres) were administered to 22 patients. Rates of PVT by branch were $5 / 22,7 / 22$, and $10 / 22$ (or $23 \%, 32 \%$ and $45 \%$ ) for left, right and main branches of the portal vein respectively. The median age of the entire cohort was 57.5 years (range, 18-78). The majority of patients were male (20 or 91\%) and Caucasian (18 or $82 \%$ ), with a small number of Asians ( 2 or $9 \%$ ) and African-Americans ( 2 or $9 \%$ ). For patients treated with resin 90Y-microspheres, all patients received their planned radiation doses with resulting near but not complete angiographic stasis and pulsatile forward flow documented at the end of each case.

Patient baseline characteristics are presented in Table 1. The average tumor burden at baseline for the entire patient cohort was significant, with 4 (18\%) of patients having $\geq 50 \%$ liver replacement by tumor, 12 (55\%) with bi-lobar disease and $3(14 \%)$ with extra-hepatic disease. Patients had varying degrees of liver dysfunction, with $7(32 \%)$ having grade 1 or higher ascites and $9(41 \%)$ with total bilirubin levels $>1.2 \mathrm{mg} / \mathrm{dL}$. These factors are reflected in Child-Pugh and CLIP score distributions of the study population. Patients generally had mild to moderate degrees of cirrhosis with 12, 6, and 1 (or 55\%, 27\% and 5\%) having Child-Pugh scores of A, $\mathrm{B}$ and $\mathrm{C}$ respectively. CLIP scores ranged from 2-5 with $18 \%$ having a CLIP score of 4 or greater. Patients with unifocal (3 or $14 \%$ ), multifocal (12 or 55\%), or infiltrative (7 or $32 \%$ ) disease were all included in the study. Three patients $(14 \%)$ were treated who had initial bilirubin levels outside of recommended values. Their bilirubin levels were 2.3, 2.5, and 7.3 $\mathrm{mg} / \mathrm{dL}$, and all three were treated with glass spheres. The patient with the highest bilirubin had a steep increase in bilirubin prior to treatment, had no other treatment options, and insisted on therapy in spite of warnings of potential toxicity.

All patients received the planned 90Y-microspheres dose at each treatment without technical complications. Fifteen (68\%) patients received only a single 90Y-microspheres treatment. Four patients underwent a total of 2 treatments and three patients underwent a total of 3 treatments. The indication for re-treatment was progressive disease. Patients with multilobar disease receiving multiple treatments all received sequential lobar treatments except for 2 cases in which whole liver treatment was done. The median interval between treatments was 98 days. The mean dose administered was $2.7 \mathrm{GBq}$, and doses ranged from 0.59 to $9.21 \mathrm{GBq}$.

The incidence of short-term (within 30 days) complications following treatment in this patient population was moderate. Table 2 lists minor and major adverse events in the 22 patients treated according to (CTCAE) version 3.0 reporting standards. The most common grade 1-2 toxicities include abdominal pain $(n=12,38 \%)$, nausea $(n=9,28 \%)$, fatigue $(n=7,22 \%)$ and anorexia $(n=6,19 \%)$. The most common grade $3-4$ toxicities include ascites $(n=4,13 \%)$, encephalopathy $(n=4,13 \%)$, fatigue $(n=3,9 \%)$ and abdominal pain $(n=3,9 \%)$. There were 4 post-treatment hospitalizations all lasting $<48$ hours. Three were for signs related to liver failure (encephalopathy and/or large volume ascites), one was due to worsening abdominal pain. One death occurred 10 days following treatment, secondary to liver failure in a patient with bilirubin outside entry criterion $(7.3 \mathrm{mg} / \mathrm{dL})$. Of the 32 treatments, no adverse event of any type was reported in $25 \%$ of treatments.

Common laboratory adverse events included increases in total bilirubin, AST and ALT levels. Total bilirubin was elevated to grade 1-2 levels following $6(19 \%)$ of the treatments and to grade 3-4 levels following $4(13 \%)$ of the treatments. AST was elevated to grade 1-2 levels following $13(41 \%)$ of the treatments and to grade 3-4 levels following $4(13 \%)$ of the 
treatments. ALT was elevated to grade 1-2 levels following $9(28 \%)$ of the treatments; no grade 3-4 elevations occurred. Of the 28 instances of grade 1-2 LFT elevation, 15 (53\%) returned to within normal limits or baseline. Of the 8 instances of grade 3-4 LFT elevation, 1 (13\%) returned to within normal limits or baseline. It should be noted that all patients experiencing grade 3-4 elevations in LFTs had pre-treatment LFTs already above the upper limits of normal.

Of the 32 treatments administered, response could be evaluated for the treated lobe in 24 treatments (15 patients). Unevaluated treatments were due to diffuse (unmeasurable) disease (found in 4 patients) or due to lack of follow up scanning (following 4 treatments, 3 secondary to patient death). Of the 24 instances of measurable disease in the treated lobe, 2 partial responses (or $8 \%$; 95\% CI: 1\%-27\%) were noted, 12 (or 50\%; 95\% CI: 29\%-71\%) resulted in stable disease, and 10 (or $42 \%$; 95\% CI: 22\%-63\%) resulted in progressive disease. For details, see Table 3 .

Of the twenty-two patients included in the analysis, thirteen have died, and nine were still alive at last follow up (see Figure 1 for a Kaplan-Meier OS plot of this cohort.) Median follow-up for survivors was approximately nine months. Five patients were alive greater than 9 months out from first treatment, and one patient was alive more than 30 months from the date of first treatment. Median overall survival was 7 months. The primary analysis cohort included three patients with bilirubin levels over $2 \mathrm{mg} / \mathrm{dL}$ at the time of therapy. When these patients were excluded from analysis, median OS increased to 8 months. When the patient with the longest survival was excluded from analysis, median OS decreased to 5 months. Patients with ChildPugh A liver disease had a significantly longer median OS of 7.7 months, versus those with Child-Pugh B and C liver disease whose median OS was 2.7 months $(p=0.01$, Figure 2). Similarly, patients with CLIP scores of 2-3 had a median OS of 7 months compared with 1.3 months for patients with CLIP scores of $4-5(\mathrm{p}=0.04$, Figure 3$)$. There was no significant difference in survival between patients with main vs. branch PVT (OS 4.4 and 7 months respectively, $\mathrm{p}=0.36$ ).

\section{DISCUSSION}

The prognosis for patients with advanced HCC complicated by PVT is poor; it has been reported that these patients have a median survival of only 2.7 to 4 months when untreated (3-5). Many treatment modalities exist for patients with HCC and PVT, however treatment benefit remains difficult to ascertain and an optimal treatment strategy has yet to emerge. PVT precludes a patient from transplantation due to the high incidence of tumor recurrence. The longest median survival times in HCC with PVT have been reported in patients able to undergo surgical resection, with studies showing median survival times of 9 to 22 months (24). Unfortunately, most patients (e.g., those with main or first branch PVT, diffuse disease, or cirrhotic change) are ineligible for surgery. Systemic therapies including chemotherapy, hormonal therapy, and interferon have shown limited benefit in this patient population (25). Recent results with sorafenib, an oral multikinase inhibitor, show promise and may represent a new standard for comparison (26). At this point, however, the survival of the subgroup of patients with significant PVT treated with sorafenib has not been reported; rather this group has been included with patients with metastatic disease. Embolic therapies such as chemoembolization or transarterial embolization, which are the mainstay for treating unresectable HCC, may be a relative contraindication in this patient population due to their limited hepatic reserve. In one publication, use of limited embolization with lipiodol and chemotherapy was tolerable, and resulted in a promising survival of 9 months (27). The patient population in this study, however, was comprised of a larger fraction of Child-Pugh A patients compared with our study. A recent study of 59 patients receiving three-dimensional conformal radiotherapy for PVT of HCC showed a 45.8\% response rate with a median survival of 10.7 
months in the responders. However, median survival for the non-responders, which comprised $54.2 \%$ of the population, was 5.3 months (28).

In contrast to chemoembolization or transarterial embolization, therapy with 90Ymicrospheres exerts less of an ischemic effect. Although embolization with 90Y-microspheres does result in permanent vascular blockade with glass or resin particles, the smaller caliber of

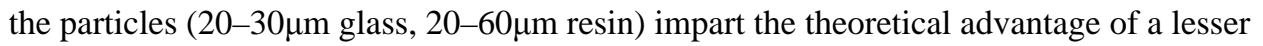
degree of macroscopic arterial occlusion. Accordingly, studies have reported a three fold lower incidence of post-embolization syndrome (abdominal pain, fever, and nausea/vomiting) following 90Y-microsphere therapy compared to chemoembolization (29). Our study shows a $38 \%$ incidence of mild abdominal pain, a $28 \%$ incidence of mild nausea and $13 \%$ incidence of mild vomiting following $90 \mathrm{Y}$-microsphere treatments. There was only a $9 \%$ incidence of severe abdominal pain following treatments, and no incidents of severe nausea/vomiting. This is comparable to the reported post-embolization syndrome incidences of $18 \%$ following glass 90Y-microsphere administration for $\mathrm{HCC}(29)$ and $25 \%$ following resin 90Y-microspheres for hepatic metastases (30). This is less than the 50\% incidence of post-embolization syndrome reported following chemoembolization in HCC with PVT (27). There were 4 hospitalizations ( $\leq 48$ hours) following therapy, 3 for complications related to liver failure and 1 due to increasing abdominal pain. Two out of 4 of these hospitalizations occurred in patients with bilirubin levels outside of entry criterion. Similarly, the one death that occurred following treatment was in a patient with bilirubin levels elevated to 7.3 prior to therapy. Median survival for our patient population was approximately 7 months (95\% CI: $4-18$ months). This is comparable to other studies of 90Y-microsphere use where median survivals of 10.0, 4.4 and 3.2 months were reported for HCC patients with branch, main and mixed (IVC, main and/or branch) PVT, respectively (13,31). Our results also fell in the same range when compared to other local non-embolic therapies such as hepatic arterial infusion chemotherapy. In a study by Takizawa, et al. examining continuous hepatic arterial infusion chemotherapy in these patients, median survival was 8.0 months (25). In a similar study of hepatic arterial infusion chemotherapy by Ikeda et al., median survival was 6.0 months (32). For details see Table 4.

Using prognostic factors as defined by the CLIP system as a basis for comparison with a historical control group, we find that the survival of our patients tended to be less than predicted. Expected median survivals for patients with CLIP scores of 2 or 3 are 13 and 7 to 8 months respectively; expected median survival for patients with CLIP scores of 4 or 5 is 2 to 3.2 months (33-34). However, it is known that PVT is an independent predictor of poor prognosis and can contribute to prognostic variability even within the same CLIP score. For example, a recent study examined intra-score variations within the CLIP system and found that median survival for patients with CLIP scores of 2 and 3 and PVT ranged from 3.5 to 6.6 months and 1.7 to 6.1 months respectively, compared to patients without PVT (6.7 to 17.9 months and 3.7 to 7.3 months respectively) (35). A similar trend is true for patients with CLIP scores of 4 and 5 . Based on that report, it does not appear as if our patients tended to fare any better or worse than predicted, based on their CLIP score. For details see Table 5.

Based on these comparisons, it is not obvious that therapy with 90Y-microspheres in patients with HCC and PVT offers a survival benefit when compared with historical controls. This would particularly apply to patients with Child-Pugh B liver disease or CLIP scores of 4 or greater who had very short survival after treatment in our series. This conclusion is of course limited by the fairly wide range of reported outcomes for this patient population, and none of the current prognostic scores seems to appropriately narrow the range of survivals for this group. One potential confounding factor is that our study included patients with extra-hepatic disease, which are frequently excluded from other studies (notably, the similar 90Ymicrosphere study by Salem et al., 12, the hepatic arterial infusion chemotherapy study by Ikeda et al., 32, and the chemoembolization study by Georgiades et al., 27.) Additional 
limitations include the retrospective nature of this and many other studies, the analysis of only acute toxicities as opposed to longer term toxicities as might occur with radiation-induced liver disease, as well as the heterogeneity of HCC patients even among the subset of those with PVT.

In conclusion, 90Y-microspheres may prolong survival beyond the median of 2.7 months reported for untreated HCC with PVT. Compared with chemoembolization, rates of severe adverse events appear low. As in other series, the one death in our series occurred in a patient with elevated bilirubin pre-treatment. Further comparison between 90Y-microspheres and systemic therapies is warranted given recent sorafenib data in an overlapping patient population.

\section{Acknowledgments}

The authors wish to thank Jennifer Jorgenson and Dr. William Dezarn for their assistance in data collection. Supported in part by NIH grant K23CA118431 and the Doris Duke Charitable Foundation Clinical Research Fellowship.

\section{REFERENCES}

(1). Parkin DM, Bray F, Ferlay J, Pisani P. Global cancer statistics, 2002. CA Cancer J Clin 2005;55:74108. [PubMed: 15761078]

(2). Pirisi M, Avellini C, Fabris C, et al. Portal vein thrombosis in hepatocellular carcinoma: age and sex distribution in an autopsy study. J Cancer Res Clin Oncol 1998;124:397-400. [PubMed: 9719503]

(3). Llovet JM, Bustamante J, Castells A, et al. Natural history of untreated nonsurgical hepatocellular carcinoma: rationale for the design and evaluation of therapeutic trials. Hepatology 1999;29:6267. [PubMed: 9862851]

(4). Yeung YP, Lo CM, Liu CL, Wong BC, Fan ST, Wong J. Natural history of untreated nonsurgical hepatocellular carcinoma. Am J Gastroenterol 2005;100:1995-2004. [PubMed: 16128944]

(5). Villa E, Moles A, Ferretti I, et al. Natural history of inoperable hepatocellular carcinoma: estrogen receptors' status in the tumor is the strongest prognostic factor for survival. Hepatology 2000;32:233-238. [PubMed: 10915729]

(6). Pentecost MJ, Daniels JR, Teitelbaum GP, Stanley P. Hepatic chemoembolization: safety with portal vein thrombosis. J Vasc Interv Radiol 1993;4:347-351. [PubMed: 8513208]

(7). Ramsey DE, Kernagis LY, Soulen MC, Geschwind JF. Chemoembolization of hepatocellular carcinoma. J Vasc Interv Radiol 2002;13:S211-S221. [PubMed: 12354839]

(8). TheraSphere. MDS Nordion; Ottawa, ON Canada: 2009. package insert

(9). SIR-Sphere. Sirtex Medical Limited; Lane Cove, NSW Australia: 2006. package insert

(10). Sato K, Lewandowski RJ, Bui JT, et al. Treatment of unresectable primary and metastatic liver cancer with yttrium-90 microspheres (TheraSphere(R)): assessment of hepatic arterial embolization. Cardiovasc Intervent Radiol 2006;29:522-529. [PubMed: 16729228]

(11). Goin JE, Dancey JE, Roberts CA, Sickles CJ, Leung DA, Soulen MC. Comparison of postembolization syndrome in the treatment of patients with unresectable hepatocellular carcinoma: trans-catheter arterial chemoembolization versus yttrium-90 glass microspheres. World J Nucl Med 2004;3:49-56.

(12). Salem R, Lewandowski R, Roberts C, et al. Use of Yttrium-90 glass microspheres (TheraSphere) for the treatment of unresectable hepatocellular carcinoma in patients with portal vein thrombosis. J Vasc Interv Radiol 2004;15:335-345. [PubMed: 15064336]

(13). Kulik LM, Carr BI, Mulcahy MF, et al. Safety and efficacy of 90Y radiotherapy for hepatocellular carcinoma with and without portal vein thrombosis. Hepatology 2008;47:71-81. [PubMed: 18027884]

(14). Kennedy AS, McNeillie P, Dezarn WA, et al. Treatment parameters and outcome in 680 treatments of internal radiation with resin $90 \mathrm{Y}$-microspheres for unresectable hepatic tumors. Int J Radiat Oncol Biol Phys 2009;74:1494-1500. [PubMed: 19157721]

J Vasc Interv Radiol. Author manuscript; available in PMC 2011 September 1. 
(15). Salem R, Thurston KG, Carr BI, Goin JE, Geschwind J-FH. Yttrium-90 microspheres: radiation therapy for unresectable liver cancer. J Vasc Interv Radiol 2002;13:S223-S229. [PubMed: 12354840]

(16). Kennedy A, Nag S, Salem R, et al. Recommendations for radioembolization of hepatic malignancies using yttrium-90 microsphere brachytherapy: a consensus panel report from the radioembolization brachytherapy oncology consortium. Int J Radiat Oncol Biol Phys 2007;68:13-23. [PubMed: 17448867]

(17). Berger MJ. Distribution of absorbed dose around point sources of electrons and beta particles in water and other media. J Nucl Med 1971:7-23. NM/MIRD Suppl 5, Pamphlet Number 7.

(18). Snyder, WS.; Ford, MR.; Warner, GG.; Watson, SB. MIRD Pamphlet Number 11. Society of Nuclear Medicine; New York, NY: S, absorbed dose per unit cumulated activity for selected radionuclides and organs; p. 1975-1976.

(19). Kennedy AS, Coldwell D, Nutting C, et al. Resin 90Y-microsphere brachytherapy for unresectable colorectal liver metastases: modern USA experience. Int J Radiat Oncol Biol Phys 2006;65:412425. [PubMed: 16690429]

(20). Salem R, Thurston KG. Radioembolization with 90yttrium microspheres: a state-of-the-art brachytherapy treatment for primary and secondary liver malignancies. Part 1: Technical and methodologic considerations. J Vasc Interv Radiol 2006;8:1251-1278. [PubMed: 16923973]

(21). Ho S, Lau WY, Leung TW, Chan M, Johnson PJ, Li AK. Clinical evaluation of the partition model for estimating radiation doses from yttrium-90 microspheres in the treatment of hepatic cancer. Eur J Nucl Med 1997;24:293-298. [PubMed: 9143467]

(22). A new prognostic system for hepatocellular carcinoma; a retrospective study of 435 patients; the Cancer of the Liver Italian Program (CLIP) investigators. Hepatology 1998;28:751-755. [PubMed: 9731568]

(23). Therasse P, Arbuck SG, Eisenhauer EA, et al. New guidelines to evaluate the response to treatment in solid tumors. European Organization for Research and Treatment of Cancer, National Cancer Institute of the United States, National Cancer Institute of Canada. J Natl Cancer Inst 2000;92:205216. [PubMed: 10655437]

(24). Minagawa M, Makuuchi M. Treatment of hepatocellular carcinoma accompanied by portal vein tumor thrombus. World J Gastroenterol 2006;12:7561-7567. [PubMed: 17171782]

(25). Takizawa D, Kakizaki S, Sohara N, et al. Hepatocellular carcinoma with portal vein tumor thrombosis: clinical characteristics, prognosis, and patient survival analysis. Dig Dis Sci 2007;52:3290-3295. [PubMed: 17394062]

(26). Simpson D, Keating GM. Sorafenib: in hepatocellular carcinoma. Drugs 2008;68:251-258. [PubMed: 18197728]

(27). Georgiades CS, Hong K, D'Angelo M, Geschwind JF. Safety and efficacy of transarterial chemoembolization in patients with unresectable hepatocellular carcinoma and portal vein thrombosis. J Vasc Interv Radiol 2005;16:1653-1659. [PubMed: 16371532]

(28). Kim DY, Park W, Lim DH, et al. Three-dimensional conformal radiotherapy for portal vein thrombosis of hepatocellular carcinoma. Cancer 2005;103:2419-2426. [PubMed: 15822130]

(29). Goin JE, Dancey JE, Roberts CA, Sickles CJ, Leung DA, Soulen MC. Comparison of postembolization syndrome in the treatment of patients with unresectable hepatocellular carcinoma: trans-catheter chemo-embolization versus yttrium-90 glass microspheres. World J Nucl Med 2004;3:49-56.

(30). Coldwell, D.; Kennedy, A. Treatment of hepatic metastases from breast cancer with yttrium-90 SIR-Spheres radioembolization. Society of Interventional Radiology 2005 annual meeting; NewOrleans, LA.

(31). Woodall CE, Scoggins CR, Ellis SF, et al. Is selective internal radioembolization safe and effective for patients with inoperable hepatocellular carcinoma and venous thrombosis? J Am Coll Surg 2009;208:375-382. [PubMed: 19317999]

(32). Ikeda M, Okusaka T, Ueno H, et al. Hepatic arterial infusion chemotherapy with epirubicin in patients with advanced hepatocellular carcinoma and portal vein tumor thrombosis. Oncology 2007;72:188-193. [PubMed: 18097170] 
(33). Farinati F, Rinaldi M, Gianni S, Naccarato R. How should patients with hepatocellular carcinoma be staged? Validation of a new prognostic system. Cancer 2000;89:2266-2273. [PubMed: 11147597]

(34). Levy I, Sherman M, Liver Cancer Study Group of the University of Toronto. Staging of hepatocellular carcinoma: assessment of the CLIP, Okuda, and Child-Pugh staging systems in a cohort of 257 patients in Toronto. Gut 2002;50:881-885. [PubMed: 12010894]

(35). Lin CY, Kee KM, Wang JH, et al. Is the Cancer of the Liver Italian Program system an adequate weighting for survival of hepatocellular carcinoma? Evaluation of intrascore prognostic value among 36 subgroups. Liver Int 2009;29:74-81. [PubMed: 18331238] 


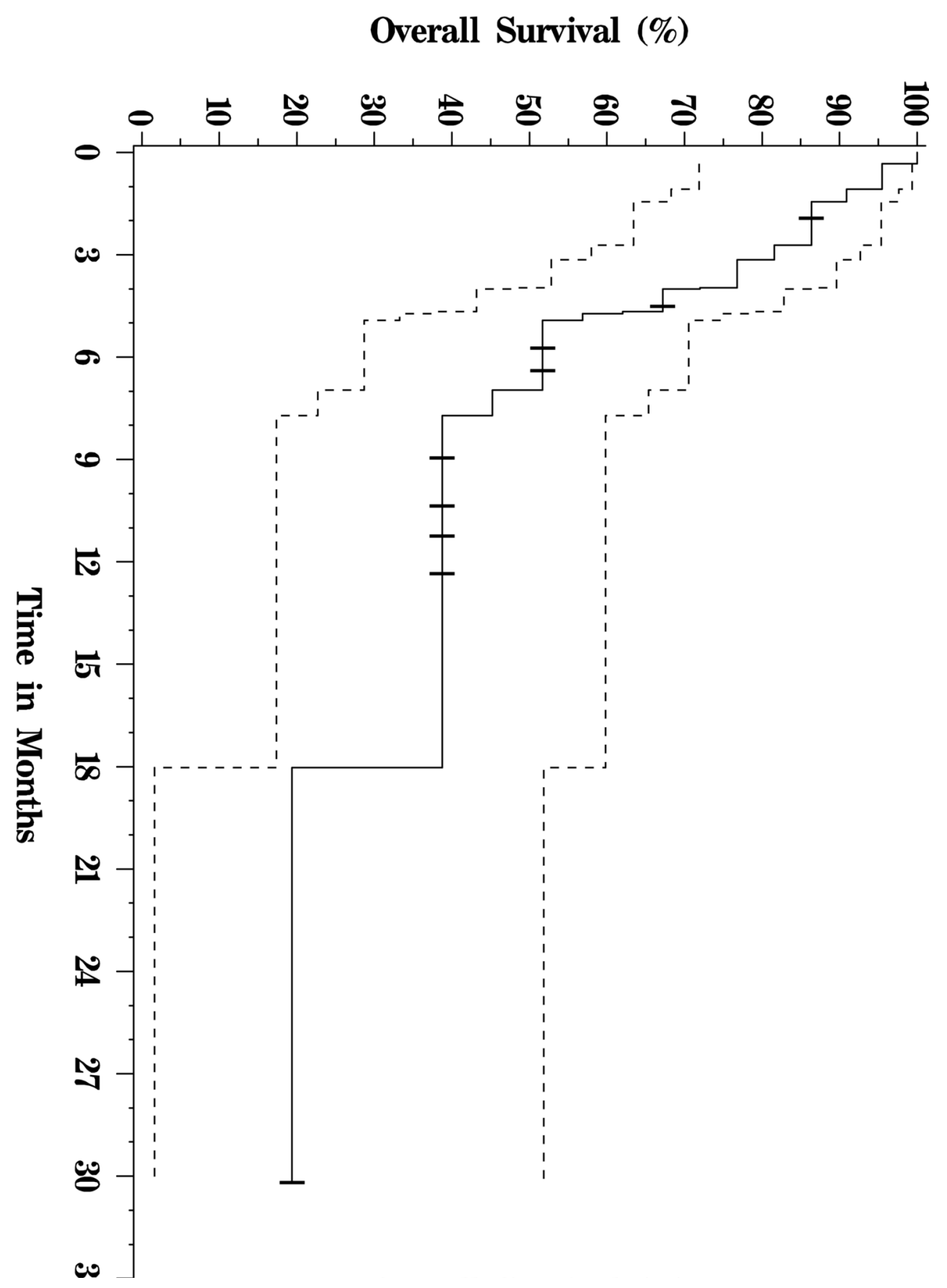

Figure 1.

Overall survival curve of 22 patients with HCC and PVT treated with 90Y-microspheres. 


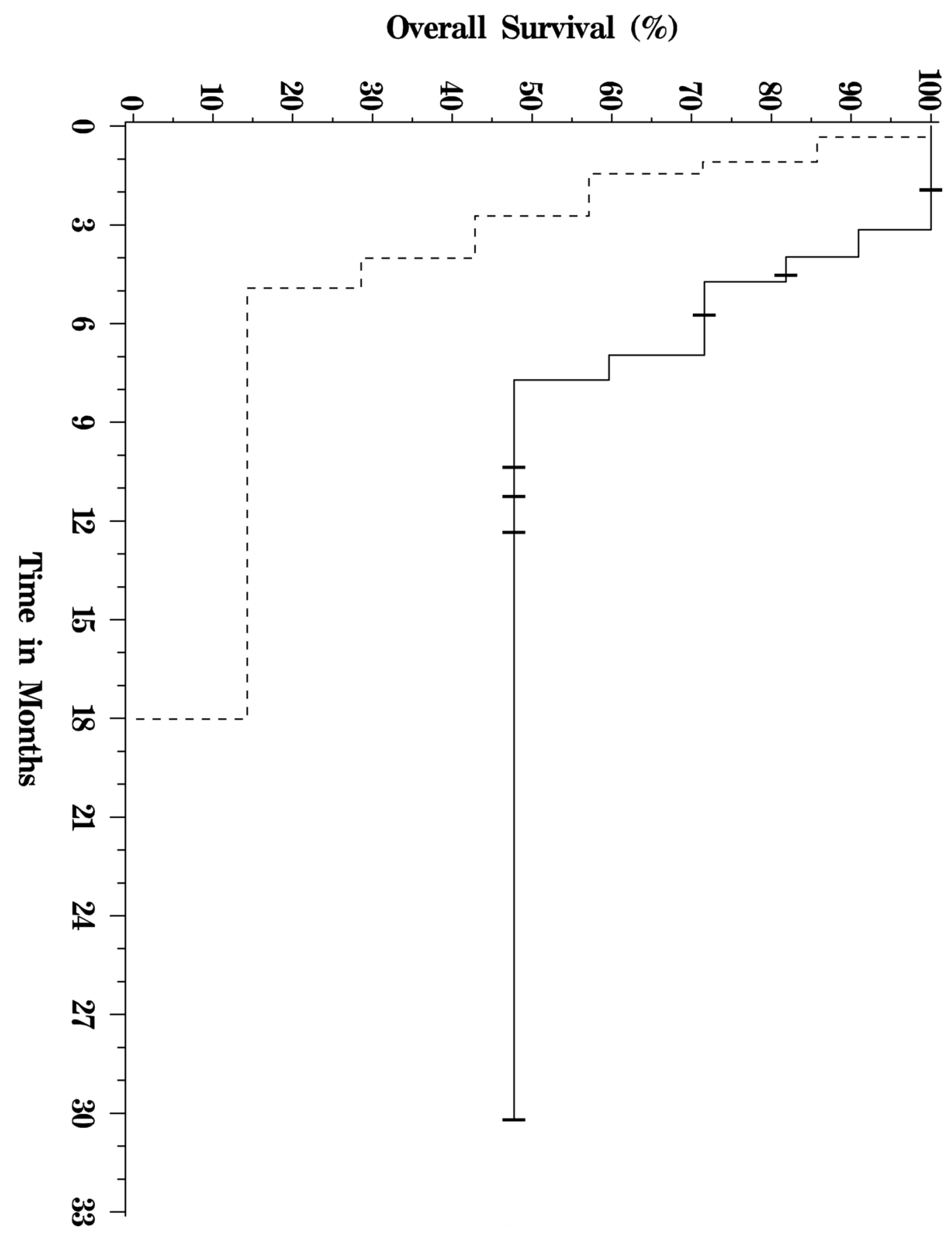

Figure 2.

Survival curve for Child-Pugh A (solid line) vs. B and C (dashed line) patients. 


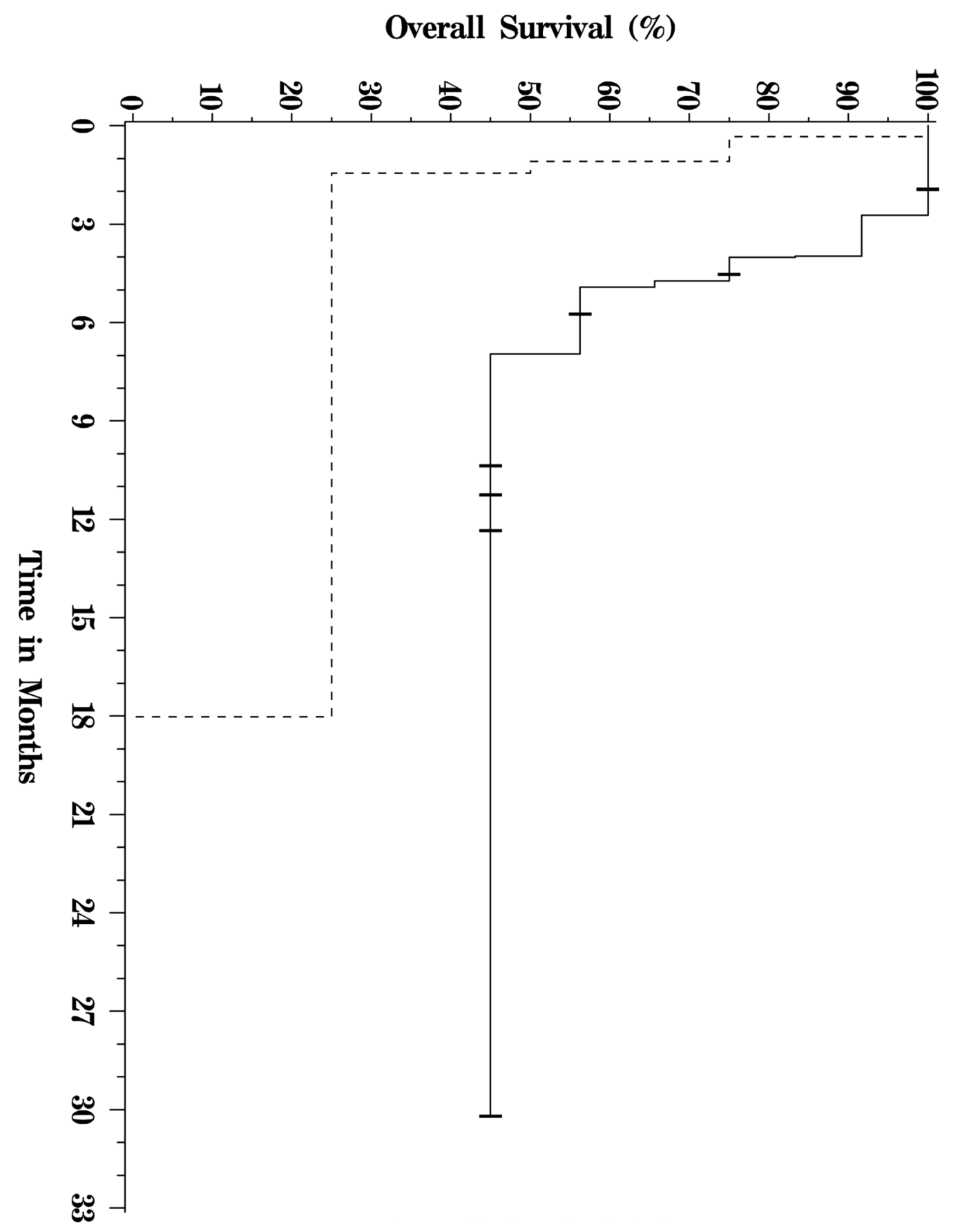

Figure 3.

Survival curve for CLIP score 2-3 (solid line) vs. 4-5 (dashed line) patients. 
Table 1

Patient characteristics prior to 90Y-microsphere treatment

\begin{tabular}{|c|c|}
\hline Characteristic & Statistic \\
\hline \multicolumn{2}{|l|}{ Age (years) } \\
\hline Median (range) & $57.5(18-78)$ \\
\hline$>65$ years, $\mathrm{n}(\%)$ & $9(41 \%)$ \\
\hline \multicolumn{2}{|l|}{ Gender, n (\%) } \\
\hline Male & $20(91 \%)$ \\
\hline Female & $2(9 \%)$ \\
\hline \multicolumn{2}{|l|}{ Extrahepatic Disease, n (\%) } \\
\hline No & $19(86 \%)$ \\
\hline Yes & $3(14 \%)$ \\
\hline \multicolumn{2}{|l|}{ Ascites, n (\%) } \\
\hline Grade 0 & $15(68 \%)$ \\
\hline Grade $1-2$ & $5(23 \%)$ \\
\hline Grade 3-4 & $2(9 \%)$ \\
\hline \multicolumn{2}{|l|}{ Serum Total Bilirubin $(\mathrm{mg} / \mathrm{dL})$} \\
\hline Median (range) & $0.85(0.1-7.3)$ \\
\hline$>1.2 \mathrm{mg} / \mathrm{dL}, \mathrm{n}(\%)$ & $9(41 \%)$ \\
\hline \multicolumn{2}{|l|}{ Tumor Distribution, n (\%) } \\
\hline Unilobar & $10(45 \%)$ \\
\hline Bilobar & $12(55 \%)$ \\
\hline \multicolumn{2}{|l|}{ Tumor Morphology, n (\%) } \\
\hline Uninodular, <50\% Replacement & $3(14 \%)$ \\
\hline Multinodular, $<50 \%$ Replacement & $15(68 \%)$ \\
\hline Massive, $\geq 50 \%$ Replacement & $4(18 \%)$ \\
\hline \multicolumn{2}{|l|}{ Child-Pugh Score, n (\%) } \\
\hline A & $12(55 \%)$ \\
\hline $\mathrm{B}$ & $6(27 \%)$ \\
\hline $\mathrm{C}$ & $1(5 \%)$ \\
\hline Unable To Be Determined & $3(14 \%)$ \\
\hline \multicolumn{2}{|l|}{ CLIP Stage, $\mathrm{n}(\%)$} \\
\hline 0 & $0(0 \%)$ \\
\hline 1 & $0(0 \%)$ \\
\hline 2 & $5(23 \%)$ \\
\hline 3 & $8(36 \%)$ \\
\hline 4 & $3(14 \%)$ \\
\hline 5 & $1(5 \%)$ \\
\hline 6 & $0(0 \%)$ \\
\hline Unable To Be Determined & $5(23 \%)$ \\
\hline
\end{tabular}




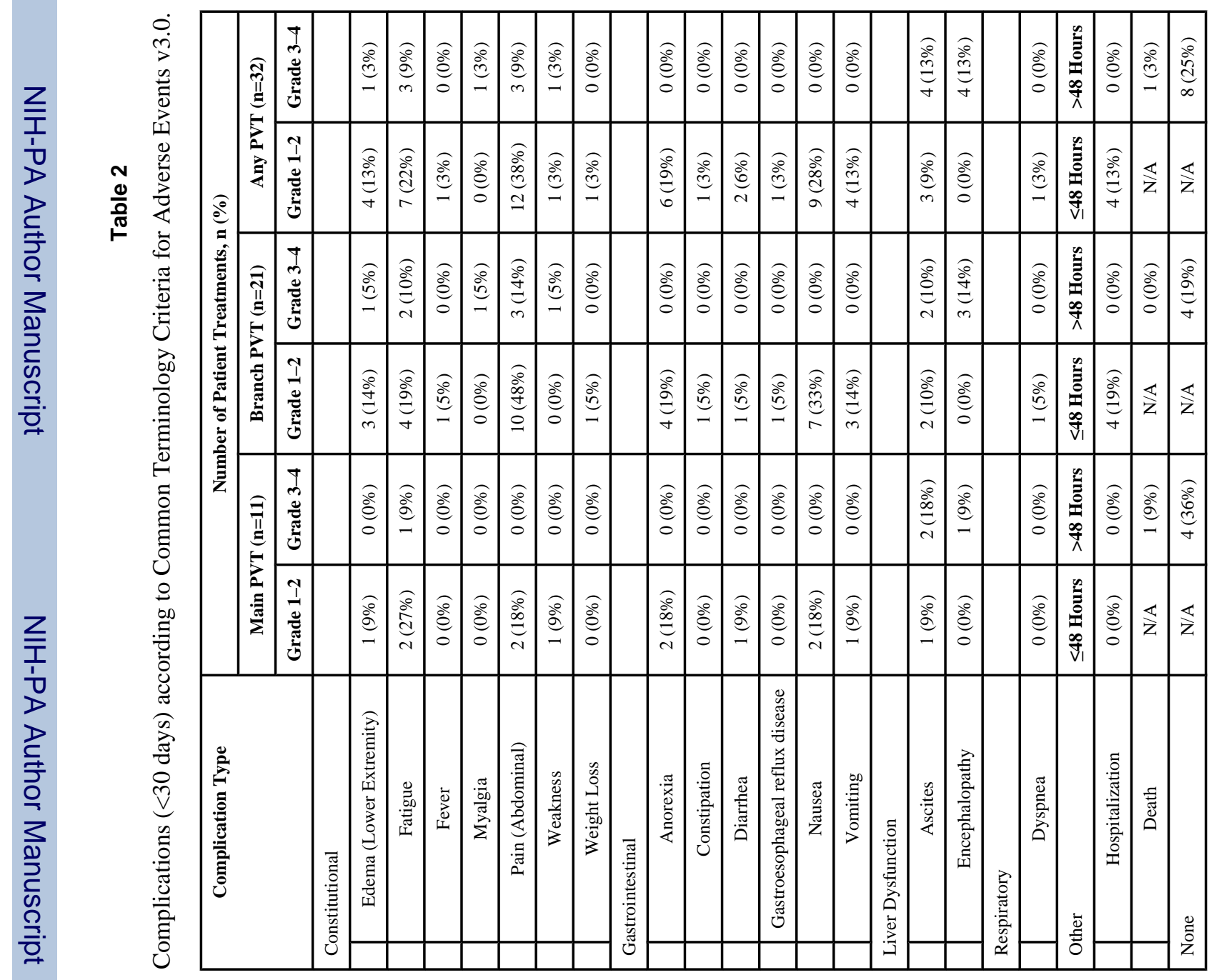




\section{Table 3}

Response to treatment.

\begin{tabular}{|l|l|l|}
\hline & \multicolumn{2}{|l|}{ Treated Lobe } \\
\hline & $\mathbf{n}(\mathbf{\%})$ & $\mathbf{9 5 \%} \mathbf{C I}^{*}$ \\
\hline Complete Response & $0(0 \%)$ & $0 \%-14 \%$ \\
\hline Partial Response & $2(8 \%)$ & $1 \%-27 \%$ \\
\hline Stable Disease & $12(50 \%)$ & $29 \%-71 \%$ \\
\hline Progressive Disease & $10(42 \%)$ & $22 \%-63 \%$ \\
\hline Total & $24(100)$ & N/A \\
\hline Unmeasurable & 4 (N/A) & N/A \\
\hline N/A & 4 (N/A) & N/A \\
\hline
\end{tabular}

* CI: exact confidence interval 
Table 4

Non-embolic trans-arterial therapies for HCC - median survival in patients with PVT.

\begin{tabular}{|l|l|l|c|c|}
\hline Treatment & PVT Type & N & Median (months) & 95\% CI* (months) \\
\hline 90Y-microspheres (31) & IVC/main/1 & 15 & 3.2 & \\
\hline 90Y-microspheres (13) & Main & 12 & 4.4 & $3-7$ \\
\hline Hepatic arterial infusion chemotherapy (32) & Main/1 & 45 & 6 & \\
\hline 90Y-microspheres (current study) & Main/1 & 22 & 7 & $4-18$ \\
\hline Continuous hepatic arterial infusion chemotherapy (25) & Main/branch & 52 & 8 & \\
\hline 90Y-microspheres (13) & Branch (1st) & 25 & 10 & $7-16$ \\
\hline
\end{tabular}

* CI: confidence interval 


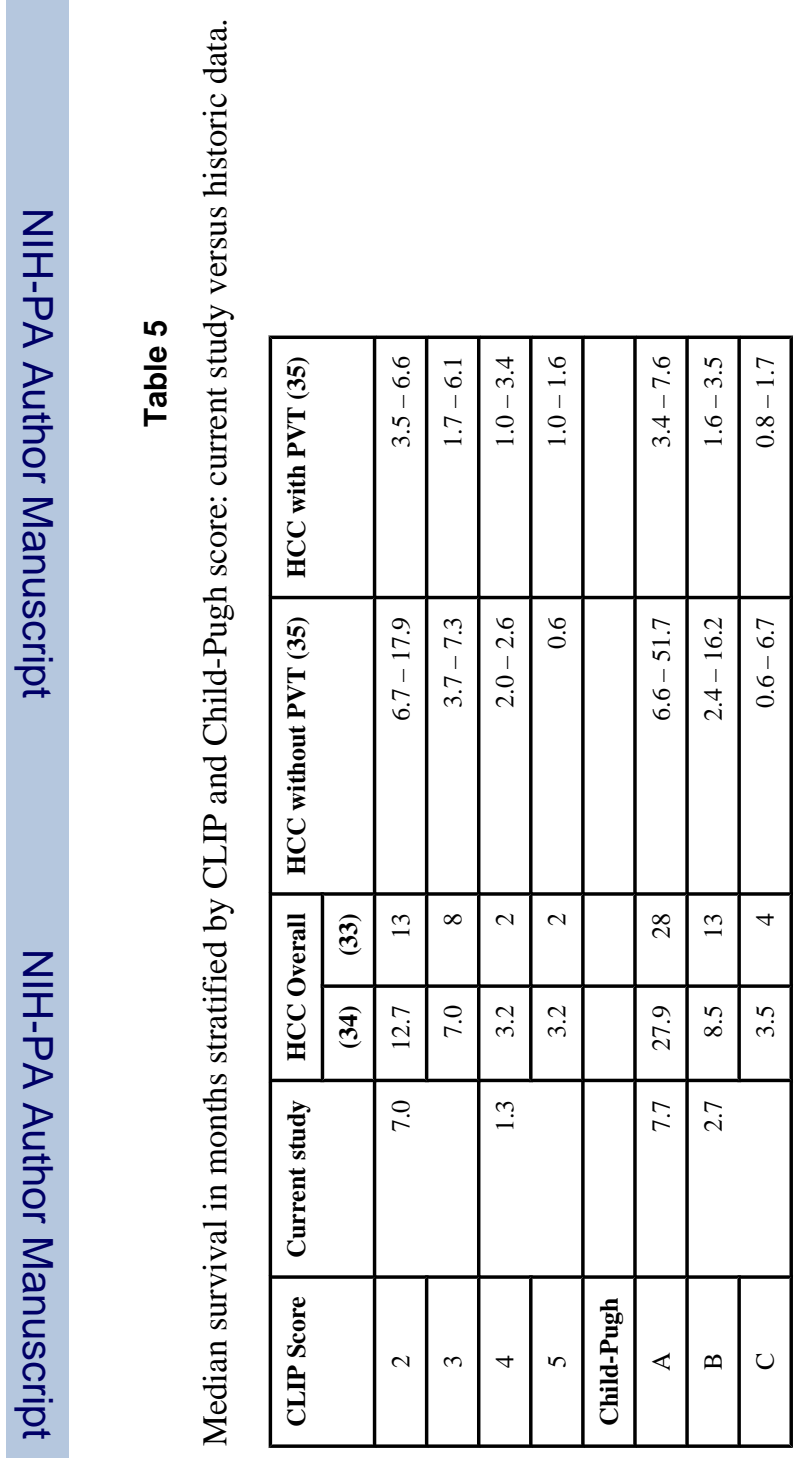

